\title{
Estudo por Imagem da Síndrome do Desfiladeiro Torácico
}

\section{Imaging Study of Thoracic Outlet Syndrome}

\author{
Marina Celli Francisco ${ }^{(1)}$, Je Hoon Yang ${ }^{(2)}$, Simone Maluf Barella ${ }^{(1)}$, Fabiano Celli Francisco ${ }^{(3)}$, \\ Jamil Natour ${ }^{(4)}$, Artur da Rocha Corrêa Fernandes ${ }^{(5)}$
}

\section{INTRODUÇÃO}

O desfiladeiro torácico consiste em dois espaços anatômicos:

1. Espaço do triângulo interescaleno: compreende o espaço entre o músculo escaleno anterior (anteriormente), músculos escalenos médio e posterior (posteriormente) e pela primeira costela (inferiormente). $\mathrm{O}$ tronco superior do plexo braquial (raízes C5 e C6) e o tronco médio (raiz C7) ocupam a porção superior deste espaço. $\mathrm{O}$ tronco inferior (raízes C8 e Tl) ocupa a parte inferior, posteriormente à artéria subclávia. A artéria subclávia situa-se na margem inferior deste espaço.

2. Espaço costoclavicular: delimitado pela metade medial da clavícula (superiormente), pela face cranial da primeira costela (inferiormente), pelo ligamento costoclavicular ou rombóide (anteriormente) e pelo músculo escaleno posterior (posteriormente).

O plexo braquial é formado pela união dos ramos ventrais das raízes C5 a C8 e a maior parte do ramo ventral de Tl. Os ramos ventrais de $\mathrm{C} 5$ e $\mathrm{C} 6$ se unem para formar o tronco superior. $\mathrm{O}$ ramo ventral de $\mathrm{C} 7$ continua como tronco médio, e os ramos ventrais de $\mathrm{C} 8 \mathrm{e} \mathrm{Tl}$ juntam-se para formar o tronco inferior. Cada um dos três troncos se divide em anterior e posterior. As divisões posteriores unem-se para formar o fascículo posterior. Já as divisões anteriores dos troncos superior e médio juntam-se para formar o fascículo lateral, e a divisão anterior do tronco inferior prossegue como fascículo medial.

O termo síndrome do desfiladeiro torácico (SDT) foi utilizado pela primeira vez por Peet et al ${ }^{1)}$, em 1956, e descreve um quadro clínico atribuído à compressão do plexo braquial, artéria e veia subclávias na região designada desfiladeiro torácico. Sua incidência varia de 3 a 80 casos/1.000 habitantes, predominando em mulheres entre 20 e 50 anos $^{(2)}$.

A SDT é dividida em cinco apresentações clínicas ${ }^{(3)}$ :
1. Neurogênica com sinais clínicos clássicos e achados compatíveis à eletroneuromiografia;

2. Neurogênica com sinais clínicos inespecíficos e sem os achados típicos à eletroneuromiografia;

3. Vascular por compressão arterial;

4. Vascular por compressão venosa;

5. Pós-traumática: apresenta sinais e sintomas de compressão neurogênica pós-traumática.

Muitas causas podem ser atribuídas à SDT, como compressão por anormalidades claviculares, costela cervical no segmento C7, alterações no primeiro arco costal, músculo subclavicular, membrana costoclavicular, ligamento costocoracóide e músculos escalenos anterior e médio ${ }^{(4)}$.

\section{QUADRO CLÍNICO}

A apresentação clínica varia com a etiologia, como mostra o diagrama a seguir:

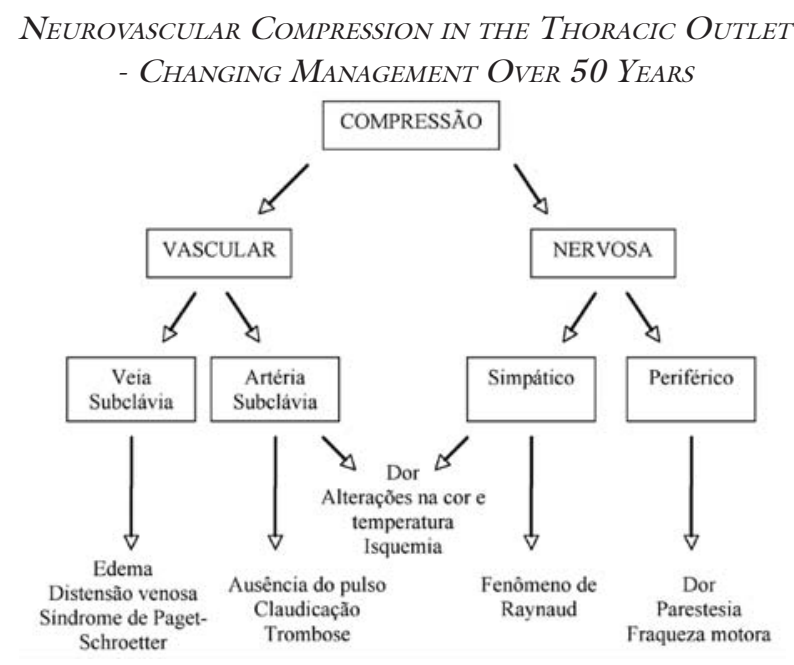

Modificado de Urschel HC Jr, Razzuk MA: Ann Surg 228(4): 609-17, 1998.

1. Médica residente de Radiologia da Universidade Federal de São Paulo (UNIFESP).

2. Mestre em Radiologia Clínica pela UNIFESP e médico colaborador do Departamento de Diagnóstico por Imagem da UNIFESP.

3. Mestre em Radiologia Clínica pela Universidade Federal do Rio de Janeiro (UFRJ) e médico radiologista do Hospital de Caridade São Brás de Porto União (SC).

4. Livre docente em Reumatologia e professor adjunto da disciplina de Reumatologia da UNIFESP.

5. Doutor em Radiologia Clínica e professor adjunto do Departamento de Diagnóstico por Imagem da UNIFESP.

Endereço para correspondência: Artur da Rocha Correa Fernandes, Rua Botucatu, 740, CEP 04023-900, São Paulo, SP, Brasil.

Rev Bras Reumatol, v. 46, n. 5, p. 353-355, set/out, 2006 
Existem cinco manobras ao exame físico que podem auxiliar no diagnóstico ${ }^{(5,6)}$ :

1. Manobra de Wright;

2. Manobra de "mãos ao alto";

3. Teste de Roos;

4. Manobra de Tinel;

5. Manobra de Adson.

Nenhum desses testes foi aceito como padrão ouro para o diagnóstico da SDT, sendo a média das manobras clínicas de $72 \%$ de sensibilidade e $53 \%$ de especificidade. A positividade das cinco manobras em pacientes com história clínica compatível confere sensibilidade e especificidade de $84 \%$. A manobra isolada mais fidedigna é a descrita por Adson $^{(6)}$.

\section{EXAMES COMPLEMENTARES}

\section{RADIOGRAFIA CONVENCIONAL}

A radiografia simples de tórax e de coluna poderá auxiliar na visualização de anormalidades ósseas, incluindo costela cervical de C7, as quais são descritas em $10 \%$ dos pacientes com SDT e entre 0,01 e $0,5 \%$ na população de uma forma geral $^{(2,5)}$ (Figura 1).

Uma possível compressão tumoral também poderá ser visualizada, embora não seja o melhor exame para se avaliar partes moles $^{(7)}$.

Segundo Gillard et $a^{\left({ }^{(6)}\right.}$, a radiografia cervical é normal em 85\% dos pacientes com quadro clínico compatível com SDT.

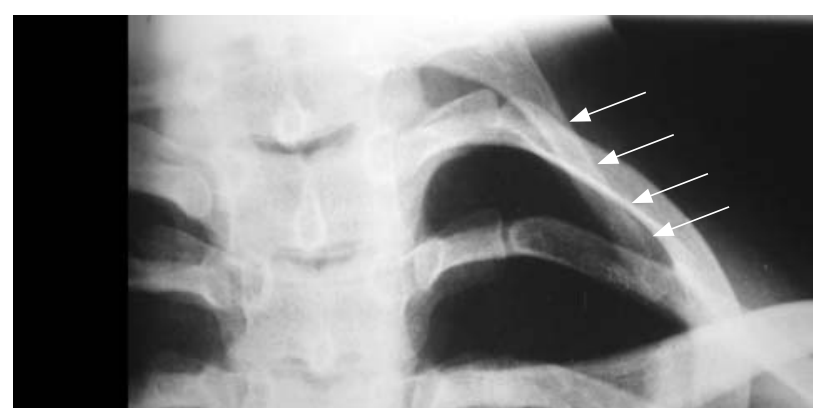

Figura 1 - Radiografia convencional da transição cervicotorácica, incidência pósteroanterior. As setas mostram uma costela cervical incompleta à esquerda.

\section{ELETRONEUROMIOGRAFIA}

A eletroneuromiografia pode ser útil na elucidação diagnóstica da SDT de origem neurogênica. No entanto, como o pinçamento nervoso é intermitente e envolve apenas um curto segmento do nervo, a eletrofisiologia, frequentemente, apresenta resultado normal. Além disso, o difícil acesso ao desfiladeiro torácico pode resultar em falsos negativos. Por estes motivos, muitos autores consideram o exame limitado na confirmação da SDT e eliminação de outros diagnósticos ${ }^{(6)}$.
O exame depende também do tipo de SDT, sua gravidade e da duração da evolução dos sintomas ${ }^{(6)}$.

\section{ANGIOGRAFIA POR SUBTRAÇÃO DIGITAL}

A angiografia é um dos métodos de imagem que podem identificar compressão arterial, mostrando o local e o tipo de estreitamento e a sua variação dinâmica com a mudança de posição do membro superior ${ }^{(8)}$ (Figura 2).

Os achados angiográficos descritos na SDT com o braço em posição neutra incluem a moderada dilatação da artéria subclávia distal/artéria axilar proximal (achado mais comum), curso anômalo da artéria subclávia distal, estenose e formação de aneurisma. Trombos murais podem estar presentes nestes aneurismas. A embolização distal é encontrada, frequentemente, em pacientes sintomáticos. Em abdução, a compressão da artéria resulta em graus variáveis de estenose e até mesmo oclusão completa ${ }^{(9)}$.

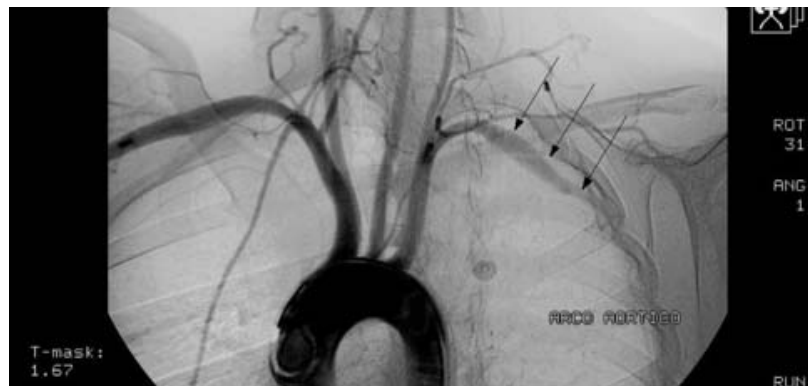

Figura 2 - Angiografia por subtração digital. Redução progressiva do calibre da artéria subclávia na projeção do espaço costoclavicular.

\section{ULTRA-SONOGRAFIA COM DOPPLER COLORIDO}

O estudo ultra-sonográfico com Doppler colorido pode detectar casos de SDT, não evidenciados às manobras clínicas. Em pacientes com suspeita clínica e duas manobras do exame físico positivas, a especificidade aumenta de 6 para $89 \%$, variando pouco a sua sensibilidade ${ }^{(6)}$.

Consiste em um exame não-invasivo e que não envolve o uso da radiação ionizante. Associado a estas vantagens, o método permite diagnosticar trombose dos vasos subclávios e pode ser utilizado quando existirem contra-indicações à angiografia ${ }^{(10)}$.

\section{ANGIOTOMOGRAFIA COMPUTADORIZADA}

A angiotomografia computadorizada é menos invasiva do que a angiografia por subtração digital, visto que utiliza apenas acesso venoso periférico. Os estudos em duas ou três dimensões podem ainda evidenciar as relações entre vasos sanguíneos e os ossos e músculos ao seu redor ${ }^{(6)}$. 
A tomografia com cortes transversos possui sensibilidade e especificidade de aproximadamente 67 e $69 \%$, respectivamente, e a reformatação sagital, de 96 e $94 \%{ }^{(11)}$. No entanto, estes dados devem ser interpretados com cuidado visto a escassez de literatura sobre o tema.

\section{RESSONÂNCIA MAGNÉTICA}

A ressonância magnética (RM) parece ser um bom método de imagem para SDT, mas ainda não existem publicações com bom nível de evidência. O exame auxilia na elucidação da causa e localização da compressão ${ }^{(12)}$ (Figura 3A).

Recentemente, séries de casos tentando realizar manobras semelhantes às do exame físico durante a execução do exame tentam mostrar benefícios em relação à ressonância em posição neutra $^{(13)}$ (Figura 3B e 3C).

Pela RM, o desfiladeiro cervical é analisado no plano sagital. A avaliação quantitativa dos espaços é dada da seguinte forma:

Espaço do triângulo interescaleno: pelo ângulo entre o músculo escaleno anterior e médio e pela maior espessura do músculo escaleno anterior. Na hiperabdução não apresentou alterações significativas.

Espaço costoclavicular: utiliza-se menor distância costoclavicular. Na hiperabdução, a clavícula desloca-se posteriormente, com compressão fisiológica da veia braquial.

Espaço retropeitoral menor: pela distância entre o músculo peitoral menor e a borda posterior da clavícula ao nível

\section{REFERÊNCIAS}

1. Peet PM, Henriksen MD, Anderson TP: Thoracic Outlet Syndrome: Evaluation of a Therapeutic Exercise Program. Mayo Clin Proc 31: 281-7, 1956.

2. Huang JH, Zager EL: Thoracic Outlet Syndrome. Neurosurgery 55: 897-903, 2004.

3. Colli BO, Carlotti CG Jr, Assirati JA Jr, Marques W Jr: Neurogenic thoracic outlet syndromes: a comparison of true and nonspecific syndromes after surgical treatment. Surg Neurol 65: 262-72, 2006.

4. Urschel HC Jr, Razzuk MA: Neurovascular Compression in the Thoracic Outlet - Changing Management Over 50 Years. Ann Surg 228: 609-17, 1998.

5. Cruz M, Matos AA, Saldanha T, Branco JC: Angiografia como método de diagnóstico da síndrome do desfiladeiro torácico neurovascular. A propósito de um caso. Rev Bras Reumatol 43: 267-71, 2003.

6. Gillard J, Pérez-Cousin M, Hachulla E, et al.: Diagnosing thoracic outlet syndrome: contribution of provocative tests, ultrasonography, electrophysiology, and helical computed tomography in 48 patients. Joint Bone Spine 68: 416-24, 2001.

7. Wright D, Jennings PR: Thoracic Outlet Syndrome. JAAPA 18: $57,2005$.

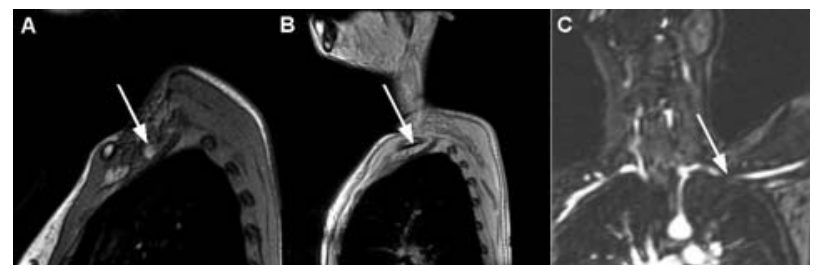

Figura 3 - Ressonância magnética, plano sagital (Figura 3A). Demonstrando hipersinal na artéria subclávia (seta) no espaço costoclavicular, que com a manobra de hiperextensão apresenta redução do calibre (Figura 3B). A angiografia por ressonância magnética da mesma paciente mostra redução do calibre da mesma na manobra de hiperextensão (Figura 3C).

da passagem dos vasos axilares. Na hiperabdução, este espaço sofre igualmente redução, rechaçando os feixes neurais contra a margem posterior do músculo peitoral menor.

\section{CONCLUSÃO}

Os exames por imagem têm papel importante no auxílio ao diagnóstico, com potencial promissor de crescimento na avaliação da SDT.

Ainda não existem diretrizes estabelecidas, mas acreditamos que para elucidar o diagnóstico devemos realizar a radiografia simples e, quando necessário, complementar o exame com a RM e exame vascular.

Declaramos a inexistência de conflitos de interesse.

8. Bogalho L, Seixas I, Martins JM, Pisco JM: Angiography in Thoracic Outlet Syndrome. Acta Med Port 11: 33-6, 1998.

9. Hagspiel KD, Spinosa DJ, Angle JF, Matsumoto AH: Diagnosis of Vascular Compression at the Thoracic Outlet Using GadoliniumEnhanced High-Resolution Ultrafast MR Angiography in Abduction and Adduction. Cardiovasc Intervent Radiol 23: 152-64, 2000.

10. Wadhwani R, Chaubal N, Sukthankar R, Shroff M, Agarwala S: Color Doppler and Duplex Sonography in 5 Patients With Thoracic Outlet Syndrome. J Ultrasound Med 20: 795-801, 2001.

11. Remy-Jardin M, Remy J, Masson P, et al.: CT Angiography of Thoracic Outlet Syndrome: Evaluation of Imaging Protocols for the Detection of Arterial Stenosis. J Comput Assist Tomograf 24: 349-61, 2000.

12. Demondion X, Bacqueville E, Paul C, Duquesnoy B, Hachulla E, Cotton A: Thoracic Outlet: Assessment with MR Imaging in Asymptomatic and Symptomatic Populations. Radiology 227: 461-8, 2003.

13. Demondion X, Boutry N, Drizenko A, Paul C, Francke JP, Cotton A: Thoracic Outlet: Anatomic Correlation with MR Imaging. AJR 175: 417-22, 2000. 